\title{
SOLUCIONES DE UN SISTEMA HIPERBÓLICO NO LINEAL CON INCLUSIÓN DE FRONTERA DIFERENCIABLE Y AMORTIGUAMIENTO DE SEGUNDO ORDEN SOBRE LA FRONTERA
}

\author{
Alfonso Pérez Salvatierra ${ }^{1}$ \\ Victoriano Yauri Luque ${ }^{2}$ \\ Zoraida J. Huamán Gutierrez ${ }^{3}$ \\ Félix Pariona Vilca ${ }^{4}$
}

Resumen.- En este artículo estudiamos la existencia de soluciones generalizadas para un sistema hiperbólico no lineal con términos discontinuos multivaluados y términos de amortiguamiento de segundo orden en la frontera.

Palabras claves: Sistemas hiperbólicos no lineales, inclusión diferenciable, amortiguamiento en la frontera, Faedo-Galerkin.

\section{ON THE SOLUTIONS OF A HYPERBOLIC NONLINEAR SYSTEM WITH BOUNDARY DIFFERENTIAL INCLUSION AND NONLINEAR SECOND ORDER DAMPING OVER THE BOUNDARY}

\begin{abstract}
In this paper we study the existence of generalized solutions for a hyperbolic nonlinear system with a discontinuous multi-valued term and non linear second-order damping terms on the boundary.

Key words: Hyperbolic nonlinear system, differential inclusion, boundary Damping, Faedo-Galerkin.
\end{abstract}

\section{Introduction}

The main purpose of this paper is to investigate the initial boundary value problem for a hyperbolic nonlinear system with differential inclusion on the boundary.

$$
\mid \begin{array}{ll}
u^{\prime \prime}-\Delta u^{\prime}-M\left(\|\nabla u\|^{2}\right) \Delta u+u^{3}=f & \text { in }(x, t) \in Q=\Omega \times(0, T) \\
u(x, 0)=u^{\prime}(x, 0)=0 & \text { in } x \in \Omega \\
u=0 & \text { on } \Sigma_{0}=\Gamma_{0} \times(0, T) \\
\frac{\partial u^{\prime}}{\partial v}+M\left(\|\nabla u\|^{2}\right) \frac{\partial u}{\partial v}+K(u) u^{\prime \prime}+\left|u^{\prime}\right|^{p} u^{\prime}+\Xi=0 & \text { on } \Sigma_{1}=\Gamma_{1} \times(0, T) \\
\Xi(x, t) \in \varphi\left(u^{\prime}(x, t)\right) & \text { a.e. }(x, t) \in \Sigma_{1}=\Gamma_{1} \times(0, T)
\end{array}
$$

\footnotetext{
${ }^{1}$ UNMSM, Facultad de Ciencias Matemáticas, Lima - Perú, e-mail:aperezs@unmsm.edu.pe ${ }^{2}$ UNMSM, Facultad de Ciencias Matemáticas, Lima - Perú, e-mail:vyauril@unmsm.edu.pe ${ }^{3}$ UNMSM, Facultad de Ciencias Matemáticas, Lima - Perú, e-mail: Zoraidahg73@hotmail.com

${ }^{4}$ UNMSM, Facultad de Ciencias Matemáticas, Lima - Perú, e-mail:parionav@unmsm.edu.pe
} 
Where $\Omega$ is a bounded open set of $\mathbb{R}^{n}(n \geq 3)$ with sufficiently smooth boundary $\Gamma=\partial \Omega$ such that $\Gamma=\Gamma_{0} \cup \Gamma_{1}, \bar{\Gamma}_{0} \cap \bar{\Gamma}_{1}=\emptyset$ and $\Gamma_{0}, \Gamma_{1}$ have positive measures, $p \in(1,+\infty)$, $M(s)$ is a $C^{1}$ class such that $M(s)>m_{0}>0$ for some constant $m_{0}, K(s)$ is a continuously differentiable positive function $\nabla u=\sum_{i=1}^{n} \frac{\partial^{2} u}{\partial x_{i}^{2}},\|\nabla u\|^{2}=\sum_{i=1}^{n} f_{\Omega}\left|\frac{\partial u}{\partial x_{i}}\right|^{2} d x, v$, is the outward unit normal vector on $\Gamma, \varphi$ is a discontinuous and nonlinear set valued mapping and $T$ is a positive real number, $u^{3}$ is a nonlinear term. The precise hypothesis on the above system will be given in the next section.

The background of these problems is in physics, especially in solid mechanics, where non-monotone and multi-valued constitutive laws lead to differential inclusion. For a brief account of the works on such variational inequalities we refer the reader to $[3,4,5]$. Motivated the results of [1], in this paper we study the existence of solutions of the variational inequalities (1.1). It is important to observe that as far as we are concerned it has never been considered differential inclusion acting on the boundary in the literature. The plan of this paper is as follow. In section 2, the assumptions and the main results are given. In section 3 , the existence of a solution to problem (1.1) is proved.

\section{Assumptions and main results}

Throughout this paper we denote

$$
\begin{array}{ll}
H_{1}(\Omega)=\left\{u \in H^{1}(\Omega) ; u=0 \text { on } \Gamma_{0}\right\} & \left.(u, v)=\int_{\Omega} u(x) v(x) d x\right) \\
(u, v)_{\Gamma_{1}}=\int_{\Gamma_{1}} u(x) v(x) d \Gamma & \|u\|_{p, \Gamma_{1}}=\left(\int_{\Gamma_{1}}|u(x)|^{p} d \Gamma\right)^{1 / p}
\end{array}
$$

For simplicity, we denote $\|u\|_{L^{2}(\Omega)}$ and $\|\cdot\|_{2, \Gamma_{1}}$ by $\|\cdot\|$ and $\|\cdot\|_{\Gamma_{1}}$ respectively. We formulate the following assumptions:

(A1) $K(s)$ is a continuous real function satisfying the conditions

$$
0<K_{0} \leq K(s) \leq K_{1}\left(1+|s|^{p}\right)
$$

$$
0 \leq\left|K^{\prime}(s)\right|^{\frac{p}{p-1}} \leq K_{2}(1+K(s))
$$

For some $K_{0}, K_{1}, K_{2}>0$ 
(A2) $b: \mathbb{R} \rightarrow \mathbb{R}$ is a locally bounded function satisfying

$$
|b(s)| \leq \mu_{1}(1+|s|) ; \forall s \in \mathbb{R}
$$

for some $\mu_{1}>0$.

The multi-valued function $\varphi: \mathbb{R} \rightarrow \mathbb{R}$ is obtained by filling in jumps of a function $b: \mathbb{R} \rightarrow \mathbb{R}$ by means of the functions $\underline{b}_{\epsilon}, \bar{b}_{\epsilon}, \underline{b}, \bar{b}: \mathbb{R} \rightarrow \mathbb{R}$ as follows:

$$
\begin{array}{cc}
\underline{b}_{\epsilon}(t)=\underset{|s-t| \leq \epsilon}{e s s \operatorname{sinf}}\{b(s)\} & \bar{b}_{\epsilon}=\underset{|s-t| \leq \epsilon}{\operatorname{ess} \sup }\{b(s)\} \\
\underline{b}(t)=\lim _{\epsilon \rightarrow 0^{+}} \underline{b}_{\epsilon}(t), & \bar{b}(t)=\lim _{\epsilon \rightarrow 0^{+}} \bar{b}_{\epsilon}(t), \varphi(t)=[\underline{b}(t), \bar{b}(t)]
\end{array}
$$

We shall use the regularization for $b$ defined by

$$
b^{m}(t)=m \int_{-\infty}^{+\infty} b(t-\tau) \rho(m \tau) d \tau
$$

Where $\rho \in C_{0}^{\infty}((-1,1)), \rho \geq 0$ and $\int_{-1}^{1} \rho(\tau)=1$.

Remark 2.1 It is easy to show that $b^{m}$ is continuous for all $m \in \mathbb{R}$ and that $\underline{b}_{\epsilon}, \bar{b}_{\epsilon}, \underline{b}, \bar{b}$, $b^{m}$ satisfy condition (A2) with a possibly different constant when $b$ satisfies (A2).

Definition: A function $u(x, t)$ such that

$$
\begin{aligned}
& u \in L^{\infty}\left(0, T ; H_{1}(\Omega)\right) \\
& u^{\prime} \in L^{2}\left(0, T ; H_{1}(\Omega)\right) \cap L^{\infty}\left(0, T ; L^{p+2}\left(\Gamma_{1}\right)\right) \\
& u^{\prime \prime} \in L^{2}\left(0, T ; H_{1}(\Omega)\right) \cap L^{2}\left(\Gamma_{1}\right)
\end{aligned}
$$

Is a generalized solution to (1.1) if exists $\Xi \in L^{2}\left(0, T ; L^{2}\left(\Gamma_{1}\right)\right)$ and for any functions 
$v \in W=H_{1}(\Omega) \cap L^{p+2}\left(\Gamma_{1}\right)$ and $\psi \in C^{1}(0, T)$ with $\psi(T)=0$ the relations hold:

$$
\mid \begin{aligned}
& \int_{0}^{T}\left\{\left(u^{\prime \prime}, v\right)+\left(\nabla u^{\prime}, \nabla v\right)+M\left(\|\nabla u\|^{2}\right)(\nabla u, \nabla v)+\right. \\
& \left.+\left(\left|u^{\prime}\right|^{p} u^{\prime}-K^{\prime}(u)\left(u^{\prime}\right)^{2}+\Xi, v\right)_{\Gamma_{1}}\right\} \psi(t) d t- \\
& +\int_{0}^{T}\left(K(u) u^{\prime \prime}, v\right)_{\Gamma_{1}} \psi^{\prime}(t) d t+ \\
& +\int_{0}^{T}\left(u^{3}(t), u^{\prime \prime}(t)\right) \psi(t)=\int_{0}^{T}(f, v) \psi(t) d t
\end{aligned}
$$

$$
\Xi(x, t) \in \varphi\left(u^{\prime}(x, t)\right) \text { a.e. }(x, t) \in \Sigma_{1}
$$

Now we are in the position to state our existence result.

Theorem: Assume que (A1) and (A2) hold and $f \in L^{2} \cdot\left(0, T ; H_{1}(\Omega)\right)$. Then, for all $T>0$ there exist a generalized solution to problem (1.1).

\section{Proof of the main theorem}

In this section we are going to show the existence of solution for problem (1.1) using the Faefo-Galenkin's approximation. For this, we represent by $\left\{w_{j}\right\}_{j \geq 1}$ a base in $W=H_{1}(\Omega) \cap L^{p+2}\left(\Gamma_{1}\right)$. Let $W_{m}=\left\langle\left\{w_{1}, w_{2}, \ldots, w_{m}\right\}\right\rangle$ subspace generate by the $m$ first vectors of the base.

We consider $u_{m}(t)=\sum_{j=1}^{m} g_{j} m(t) w$; the solution of the problem approaching of Cauchy:

$$
\begin{gathered}
\mid \begin{array}{c}
\left(u_{m}^{\prime \prime}(t), w_{j}\right)+\left(\nabla u_{m}^{\prime}, \nabla w_{j}\right)+M\left(\left|\nabla u_{m}\right|^{2}\right)\left(\nabla u_{m}, \nabla w_{j}\right)+ \\
\left(K\left(u_{m}\right) u_{m}^{\prime \prime}+\left|u_{m}^{\prime}\right|^{p} u_{m}^{\prime}+b^{m}\left(u_{m}^{\prime}\right), w_{j}\right)_{\Gamma_{1}}+\left(u_{m}^{3}, w_{j}\right)=\left(f(t), w_{j}\right) ; \forall w_{j} \in W_{m}
\end{array} \\
u_{m}(0)=u_{m}^{\prime}(0)=0
\end{gathered}
$$

By the theorem of Caratheodory, the, approximate system (3.1) and (3.2) has solutions $u_{m}(t)$ in $\left[0, t_{m}\right\rangle$, to see [6].

The extension of these solutions to the whole interval $[0, T]$ is a consequence of the priori estimate which we are going to prove below. 


\section{STEP 1: A PRIORI ESTIMATE}

Multiplying (3.1) by $g_{j}^{\prime} m(t)$ and summing from $j=1$ to $j=m$, and definition of $u_{m}$, we get.

$$
\begin{aligned}
& \left(u_{m}^{\prime \prime}(t), u_{m}^{\prime}(t)\right)+\left(\nabla u_{m}^{\prime}(t), \nabla u_{m}^{\prime}(t)\right)+M\left(\left|\nabla u_{m}(t)\right|^{2}\right)\left(\nabla u_{m}(t), \nabla u_{m}^{\prime}(t)\right)+ \\
& \left(K\left(u_{m}(t)\right) u_{m}^{\prime \prime}(t)+\left|u_{m}^{\prime}(t)\right|^{p} u_{m}^{\prime}(t)+b^{m}\left(u_{m}^{\prime}(t), u_{m}^{\prime}\right)\right)_{\Gamma_{1}}+\left(u_{m}^{3}(t), u_{m}^{\prime}(t)\right)=\left(f(t), u_{m}^{\prime}(t)\right)
\end{aligned}
$$

From where we obtain

$$
\begin{aligned}
& \frac{1}{2} \frac{d}{d t}\left\{\left\|u_{m}^{\prime}(t)\right\|^{2}+\bar{M}\left(\left\|\nabla u_{m}(t)\right\|^{2}\right)+\int_{\Gamma_{1}} K\left(u_{m}(x, t)\right)\left(u_{m}^{\prime}(x, t)\right)^{2} d \Gamma+\frac{1}{2}\left\|u_{m}(t)\right\|_{4}^{4}\right\}+ \\
& +\int_{\Gamma_{1}} b^{m}\left(u_{m}^{\prime}(x, t)\right) u_{m}^{\prime}(x, t) d \Gamma+\left\|\nabla u_{m}^{\prime}(t)\right\|^{2}+\left\|u_{m}^{\prime}(t)\right\|_{p+2}^{p+2}+ \\
& +\frac{1}{2} \int_{\Gamma_{1}} K^{\prime}\left(u_{m}(x, t)\right)\left(u_{m}^{\prime}(x, t)\right)^{3} d \Gamma=\left(f(t), u_{m}^{\prime}(t)\right)
\end{aligned}
$$

Where $\bar{M}(s)=\int_{0}^{s} M(r) d r$.

Therefore, integrating over $(0, t)$ and $u_{m}(0)=u_{m}^{\prime}(0)=0$,

$$
\begin{aligned}
& \frac{1}{2}\left\{\left\|u_{m}^{\prime}(t)\right\|^{2}+\bar{M}\left(\left\|\nabla u_{m}(t)\right\|^{2}\right)+\frac{1}{2}\left\|u_{m}(t)\right\|_{4}^{4}\right. \\
& \left.\int_{\Gamma_{1}} K\left(u_{m}(x, t)\right)\left(u_{m}^{\prime}(x, t)\right)^{2} d \Gamma\right\}+\int_{0}^{t}\left\|\nabla u_{m}^{\prime}(s)\right\|^{2} d s \\
& \int_{0}^{t}\left\|u_{m}^{\prime}(s)\right\|_{p+2, \Gamma_{1}}^{p+2} d s+\int_{0}^{t} \int_{\Gamma_{1}} b^{m}\left(u_{m}^{\prime}(x, s)\right) u_{m}^{\prime}(x, s) d \Gamma d s- \\
& +\frac{1}{2} \int_{0}^{t} \int_{\Gamma_{1}} K^{\prime}\left(u_{m}(x, s)\right)\left(u_{m}^{\prime}(x, s)\right)^{3} d \Gamma d s=\int_{0}^{t}\left(f(s), u_{m}^{\prime}(s)\right) d s
\end{aligned}
$$


For the condition of (A2) we have

$$
\begin{aligned}
\left\|b^{m}\left(u_{m}^{\prime}(t)\right)\right\|_{\Gamma_{1}}^{2} & \left.=\int_{\Gamma_{1}}\left(b^{m}\left(u_{m}^{\prime}(x, t)\right)\right)\right)^{2} d \Gamma \\
& \leq \int_{\Gamma_{1}} c_{1}\left(1+u_{m}^{\prime}(x, t)\right)^{2} d \Gamma \\
& \leq 2 c_{1} \int_{\Gamma_{1}} c_{1}\left(1+\left|u_{m}^{\prime}(x, t)\right|^{2}\right) d \Gamma \\
& \leq c_{2}+2 c_{1}\left\|u_{m}^{\prime}(t)\right\|_{\Gamma_{1}}^{2}
\end{aligned}
$$

From (3.4) and by the Holder's inequality

$$
\begin{aligned}
& \left|\int_{0}^{t}\left(b^{m}\left(u_{m}^{\prime}(s), u_{m}^{\prime}(s)\right)\right)_{\Gamma_{1}} d s\right| \\
& \leq\left(\int_{0}^{t}\left\|b^{m}\left(u_{m}^{\prime}(s)\right)\right\|_{\Gamma_{1}}^{2} d s\right)^{1 / 2}\left(\int_{0}^{t}\left\|u_{m}^{\prime}(s)\right\|_{\Gamma_{1}}^{2} d s\right)^{1 / 2} \\
& \leq\left(\int_{0}^{t}\left(c_{2}+2 c_{1}\left\|u_{m}^{\prime}(s)\right\|_{\Gamma_{1}}^{2}\right) d s\right)^{1 / 2}\left(\int_{0}^{t}\left\|u_{m}^{\prime}(s)\right\|_{\Gamma_{1}}^{2} d s\right)^{1 / 2} \\
& \leq c_{3}\left(1+\int_{0}^{t}\left\|u_{m}^{\prime}(s)\right\|_{\Gamma_{1}}^{2} d s\right)^{1 / 2}
\end{aligned}
$$

Let us observe that, by Young's inequality

$$
\begin{aligned}
& \int_{0}^{t}\left\{\left\|u_{m}^{\prime}(s)\right\|_{p+2, \Gamma_{1}}^{p+2}-\frac{1}{2} \int_{\Gamma_{1}} K^{\prime}\left(u_{m}(s)\right)\left(u_{m}^{\prime}(s)\right)^{3} d \Gamma\right\} d s \geq \\
& \int_{0}^{t} \int_{\Gamma_{1}}\left|u_{m}^{\prime}(s)\right|^{2}\left\{\left|\dot{u}_{m}^{\prime}(s)\right|^{p}-\epsilon\left|u_{m}^{\prime}(s)\right|^{p}-c(\epsilon)\left|K^{\prime}\left(u_{m}(s)\right)\right|^{\frac{p}{p-1}}\right\} d \Gamma d s
\end{aligned}
$$

Also we notice that,

$$
\int_{0}^{t}\left|f(s)\left\|u_{m}^{\prime}(s) \mid d s \leq \int_{0}^{t}\right\| f(s)\left\|^{2} d s+\int_{s}^{t}\right\| u_{m}^{\prime}(s) \|^{2} d s\right.
$$


From (3.5), (3.6), (3.7) and for $\epsilon=\frac{1}{2}$ we obtain

$$
\begin{aligned}
& \frac{1}{2}\left\{\left\|u_{m}^{\prime}(t)\right\|^{2}+\bar{M}\left(\left\|\nabla u_{m}(t)\right\|^{2}\right)+\frac{1}{2}\left\|u_{m}(t)\right\|_{4}^{4}+\int_{\Gamma_{1}} K\left(u_{m}(t)\right)\left(u_{m}^{\prime}(t)\right)^{2} d \Gamma\right\}+ \\
& +\int_{0}^{t}\left\|\nabla u_{m}^{\prime}(s)\right\|^{2} d s+\frac{1}{2} \int_{0}^{t} \int_{\Gamma_{1}}\left|u_{m}^{\prime}(s)\right|^{p+2} d \Gamma d s \\
& \leq c(\epsilon) \int_{0}^{t} \int_{\Gamma_{1}}\left|u_{m}^{\prime}(s)\right|^{2}\left|K^{\prime}\left(u_{m}(s)\right)\right|^{\frac{p}{p-1}} d \Gamma d s \\
& \leq c_{3}\left(1+\int_{0}^{t}\left\|u_{m}^{\prime}(s)\right\|_{\Gamma_{1}}^{2} d s\right)+\int_{0}^{t}\|f(s)\|^{2} d s+\int_{0}^{t}\left\|u_{m}^{\prime}(s)\right\|^{2} d s
\end{aligned}
$$

On the other hand, we observe that:

$$
K(u) \geq c_{0}(1+K(u)) \text { where } 2 c_{0}=\min \left\{1, K_{0}\right\}
$$

from where

$$
\int_{\Gamma_{1}}\left|u_{m}^{\prime}(t)\right|^{2}\left|K\left(u_{m}(t)\right)\right| d \Gamma \geq c_{0} \int_{\Gamma_{1}}\left|u_{m}^{\prime}(t)\right|^{2}\left|1+K\left(u_{m}(t)\right)\right| d \Gamma
$$

Also, from (2.2)

$$
\left|K^{\prime}(s)\right|_{p-1}^{p} \leq K_{2}(1+K(s))
$$

Then

$$
\begin{aligned}
& c(\epsilon) \int_{0}^{t} \int_{\Gamma_{1}}\left|u_{m}^{\prime}(x, s)\right|^{2}\left|K^{\prime}\left(u_{m}(x, s)\right)\right|^{\frac{p}{p-1}} d \Gamma d s \leq \\
& \leq c(\epsilon) K_{2} \int_{0}^{t} \int_{\Gamma_{1}}\left|u_{m}^{\prime}(x, s)\right|^{2}\left|1+K\left(u_{m}(x, s)\right)\right| d \Gamma d s
\end{aligned}
$$


From (3.9) and (3.10) majority to right and left in (3.8)

$$
\begin{aligned}
& \frac{1}{2}\left\{\left\|u_{m}^{\prime}(t)\right\|^{2}+\bar{M}\left(\left\|\nabla u_{m}(t)\right\|^{2}\right)+\frac{1}{2}\left\|u_{m}(t)\right\|_{4}^{4}+\right. \\
& \left.+c_{0} \int_{\Gamma_{1}}\left|u_{m}^{\prime}(t)\right|^{2}\left[1+K\left(u_{m}(t)\right)\right] d \Gamma\right\}+\int_{0}^{t}\left\|\nabla u_{m}^{\prime}(s)\right\|^{2} d s+ \\
& +\frac{1}{2} \int_{0}^{t}\left\|u_{m}^{\prime}(s)\right\|_{p+2, \Gamma_{1}}^{p+2} d s \leq \\
& \leq c \int_{0}^{t} \int_{\Gamma_{1}}\left|u_{m}^{\prime}(x, s)\right|^{2}\left[1+K\left(u_{m}(x, s)\right)\right] d \Gamma d s+ \\
& +c_{3}\left(1+\int_{0}^{t} \int_{\Gamma}\left|u_{m}^{\prime}(x, s)\right|^{2} d \Gamma d s\right)+\int_{0}^{t}\|f(s)\|^{2} d s+ \\
& +\int_{0}^{t}\left|u_{m}^{\prime}(s)\right|^{2} d s ; c=c(\epsilon)+K_{2}
\end{aligned}
$$

Let us define,

$$
\begin{aligned}
E_{m}(t)= & \frac{1}{2}\left\{\left\|u_{m}^{\prime}\right\|^{2}+\bar{M}\left(\left\|\nabla u_{m}(t)\right\|^{2}\right)+\frac{1}{2}\left\|u_{m}(t)\right\|_{4}^{4}\right\}+ \\
& +c_{0} \int_{\Gamma_{1}}\left|u_{m}^{\prime}(t)\right|^{2}\left[1+K\left(u_{m}(t)\right)\right] d \Gamma
\end{aligned}
$$

then, of (3.11) definition of $E_{m}$ we obtain

$$
E_{m}(t) \leq c_{4}\left(1+\int_{0}^{t} E_{m}(s) d s\right)
$$

Thus, by Gronwall's lemma, we conclude that

$$
E_{m}(t) \leq c_{5} ; \forall t \in[0, T]
$$

From (3.11) and (3.12) it is obtained $\forall t \in[0, T]$

$$
\int_{0}^{t}\left\|\nabla u_{m}^{\prime}(s)\right\|^{2} d s \leq c_{6}, \int_{\Gamma_{1}}\left\|u_{m}^{\prime}(t)\right\|^{2} d \Gamma \leq c_{\tau}
$$

By imbedding theorem and from (3.13) we have

$$
\int_{0}^{t}\left\|u_{m}^{\prime}(s)\right\|^{2} d s \leq c_{8}
$$


Furthermore, from (3.4) y (3.14) we obtain

$$
\int_{0}^{t}\left\|b^{m}\left(u_{m}^{\prime}(s)\right)\right\|_{\Gamma_{1}}^{2} d s \leq c_{9}
$$

Since $\bar{M}\left(\left\|\nabla u_{m}(t)\right\|^{2}\right) \geq m_{0}\left\|\nabla u_{m}(t)\right\|^{2}$, by $(3.12)$

$$
\left\|\nabla u_{m}(t)\right\|^{2} \leq c_{10}
$$

Similarly from (3.12) we obtain

$$
\left\|u_{m}(t)\right\|_{4}^{4} \leq c_{11}
$$

From (3.17) we can say

$$
\left(u_{m}\right) \text { is bounded in } L^{\infty}\left(0, T ; L^{4}(\Omega)\right)
$$

Applying the theorem of Aubin-Lions $B_{0}=H_{1}(\Omega), B=B_{1}=L^{2}(\Omega)$ and $p_{0}=2=p_{1}$, we can obtain a denoted subsuccesion in the same way

$$
u_{m}^{3} \rightarrow u^{3} \text { a.e. in } Q
$$

From (3.18) we conclude that $\left(u_{m}^{3}\right)$ is bounded in $L^{4 / 3}(Q)$ of where

$$
\begin{gathered}
u_{m}^{3} \rightarrow u^{3} \text { weak in } L^{4 / 3}(Q)=\left[L^{4}(Q)\right]^{\prime}, \text { i.e., } \\
\int_{0}^{T}\left(u_{m}^{3} \cdot w_{j}\right) \psi(t) d t \rightarrow \int_{0}^{T}\left(u^{3}, w_{j}\right) \psi(t) d t, \psi \in C^{1}(0, T)
\end{gathered}
$$

Multiplying (3.1) by $g_{j m}^{\prime \prime}(t)$ and summing from $j=1$ to $j=m$ and definition from $u_{m}(t)$, we have

$$
\begin{aligned}
& \left\|u_{m}^{\prime \prime}(t)\right\|^{2}+\frac{1}{2} \frac{d}{d t}\left\|\nabla u_{m}^{\prime}(t)\right\|^{2}+M\left(\left\|\nabla u_{m}(t)\right\|^{2}\right) \frac{d}{d t}\left(\nabla u_{m}(t), \nabla u_{m}^{\prime}(t)\right)- \\
& +M\left(\left\|\nabla u_{m}(t)\right\|^{2}\right)\left\|\nabla u_{m}^{\prime}(t)\right\|^{2}+\left(b^{m}\left(u_{m}^{\prime}(t), u_{m}^{\prime}(t)\right)\right)_{\Gamma_{1}}+\int_{\Gamma_{1}} K\left(u_{m}(t)\right)\left(u_{m}^{\prime \prime}(t)\right)^{2} d \Gamma+ \\
& +\frac{1}{p+2} \frac{d}{d t}\left\|u_{m}^{\prime}(t)\right\|_{p+2, \Gamma_{1}}^{p+2}+\int_{\Omega} u_{m}^{3}(x) u_{m}^{\prime \prime}(x) d x=\left(f(t), u_{m}^{\prime \prime}(t)\right)
\end{aligned}
$$


Integrating this equality over $(0, t)$

$$
\begin{aligned}
& \int_{0}^{t}\left\|u_{m}^{\prime \prime}(t)\right\|^{2} d s+\frac{1}{2} \int_{0}^{t} \frac{d}{d s}\left\|\nabla u_{m}^{\prime}(s)\right\|^{2} d s+ \\
& +\int_{0}^{t} M\left(\left\|\nabla u_{m}(s)\right\|^{2}\right) \frac{d}{d s}\left(\nabla u_{m}(t), \nabla u_{m}^{\prime}(t)\right) d s- \\
& +\int_{0}^{t} M\left(\left\|\nabla u_{m}(s)\right\|^{2}\right)\left\|\nabla u_{m}^{\prime}(t)\right\|^{2} d s+ \\
& +\int_{0}^{t} \int_{\Gamma_{1}} K\left(u_{m}(s)\right)\left(u_{m}^{\prime \prime}(s)\right)^{2} d \Gamma d s+\int_{0}^{t} \int_{\Gamma_{1}} b^{m}\left(u_{m}^{\prime}(x, s), u_{m}^{\prime \prime}(x, s)\right) d \Gamma d s+ \\
& +\frac{1}{p+2} \int_{0}^{t} \frac{d}{d s}\left\|u_{m}^{\prime}(s)\right\|_{p+2, \Gamma_{1}}^{p+2} d s+\int_{0}^{t} \int_{\Omega} f(x, s) d x d s \\
& +\int_{0}^{t} \int_{\Omega}^{3} u_{m}^{3}(x, s) u_{m}^{\prime \prime}(x, s) d x d s=\int^{2}
\end{aligned}
$$

Note that, from

$$
\int_{0}^{t} \int_{\Gamma_{1}} K\left(u_{m}(x, s)\right)\left(u_{m}^{\prime \prime}(x, s)\right)^{2} d \Gamma d s \geq K_{0} \int_{0}^{t}\left\|u_{m}^{\prime \prime}(s)\right\|_{\Gamma_{1}}^{2} d s
$$

On the other hand, we note what

$$
\frac{1}{p+2} \int_{0}^{t} \frac{d}{d s}\left\|u_{m}^{\prime}(s)\right\|_{p+2, \Gamma_{1}}^{p+2} d s=\frac{1}{p+2}\left\|u_{m}^{\prime}(t)\right\|_{o+2, \Gamma_{1}}^{p+2}
$$

and

$$
\begin{array}{r}
\int_{0}^{t} M\left(\left\|\nabla u_{m}(s)\right\|^{2}\right) \frac{d}{d s}\left(\nabla u_{m}(t), \nabla u_{m}^{\prime}(t)\right) d s=M\left(\left\|\nabla u_{m}(t)\right\|^{2}\right)\left(\nabla u_{m}(t), \nabla u_{m}^{\prime}(t)\right)- \\
2 \int_{0}^{t} M^{\prime}\left(\left\|\nabla u_{m}(s)\right\|^{2}\right)\left(\nabla u_{m}(s), \nabla u_{m}^{\prime}(s)\right)^{2} d s
\end{array}
$$


Next, this in (3.21)

$$
\begin{aligned}
& \int_{0}^{t}\left\|u_{m}^{\prime \prime}(t)\right\|^{2} d s+\frac{1}{2}\left\|\nabla u_{m}^{\prime}(t)\right\|^{2}+ \\
& M\left(\left\|\nabla u_{m}(t)\right\|^{2}\right)\left(\nabla u_{m}(t), \nabla u_{m}^{\prime}(t)\right)- \\
& 2 \int_{0}^{t} M^{\prime}\left(\left\|\nabla u_{m}(s)\right\|^{2}\right)\left(\nabla u_{m}(s), \nabla u_{m}^{\prime}(s)\right)^{2} d s+ \\
& K_{0} \int_{0}^{t}\left\|u_{m}^{\prime \prime}(s)\right\|_{\Gamma_{1}}^{2} d s+\frac{1}{p+2}\left\|u_{m}^{\prime}(t)\right\|_{p+2, \Gamma_{1}}^{p+2} \leq \\
& \leq \int_{0}^{t} M\left(\left\|\nabla u_{m}(s)\right\|^{2}\right)\left\|\nabla u_{m}^{\prime}(s)\right\|^{2} d s- \\
& +\int_{s}^{t} \int_{\Gamma_{1}} b^{m}\left(u_{m}^{\prime}(x, s)\right) u_{m}^{\prime \prime}(x, s) d \Gamma d s- \\
& +\int_{0}^{t} \int_{\Omega} u_{m}^{3}(x, s) u_{m}^{\prime \prime}(x, s) d x d s+\int_{0}^{t} \int_{\Omega} f(x, s) u_{m}^{\prime \prime}(x, s) d x d s
\end{aligned}
$$

By Young's inequality, and immersion $H_{0}^{1}(\Omega) \rightarrow L^{4}(\Omega)$ :

$$
\left\{\begin{array}{l}
-\int_{0}^{t} \int_{\Gamma_{1}} b^{m}\left(u_{m}^{\prime}(x, s)\right) u_{m}^{\prime \prime}(x, s) d \Gamma d s \leq \\
\leq c(\epsilon) \int_{0}^{t}\left\|b^{m}\left(u_{m}^{\prime}(s)\right)\right\|_{\Gamma_{1}}^{2} d s+\epsilon \int_{0}^{t}\left\|u_{m}^{\prime \prime}(s)\right\|_{\Gamma_{1}}^{2} d s- \\
+\int_{0}^{t} \int_{\Omega}^{3} u_{m}^{3}(x, s) u_{m}^{\prime \prime}(x, s) d x d s \leq \\
\leq c \int_{0}^{t}\left\|u_{m}(s)\right\|_{H_{0}^{1}}^{4} d s+\widetilde{c} \int_{0}^{t}\left\|u_{m}^{\prime \prime}(s)\right\|_{H_{0}^{1}}^{4} d s<c_{12} \\
\int_{0}^{t} \int_{\Omega}^{t} f(x, s) u_{m}^{\prime \prime}(x, s) d x d s \leq \\
\leq \epsilon \int_{0}^{t}\left\|u_{m}^{\prime \prime}(s)\right\|^{2} d s+c(\epsilon) \int_{0}^{t}\|f(s)\|^{2} d s
\end{array}\right.
$$

From (3.23) in (3.22) we have

$$
\begin{aligned}
& \int_{0}^{t}\left\|u_{m}^{\prime \prime}(s)\right\|^{2} d s+\frac{1}{2}\left\|\nabla u_{m}^{\prime}(t)\right\|^{2}+K_{0} \int_{0}^{t}\left\|u_{m}^{\prime \prime}(s)\right\|_{\Gamma_{1}}^{2} d s+ \\
& +\frac{1}{p+2}\left\|u_{m}^{\prime}(t)\right\|_{p+2, \Gamma_{1}}^{p+2} \leq-M\left(\left\|\nabla u_{m}(t)\right\|^{2}\right)\left(\nabla u_{m}(t), \nabla u_{m}^{\prime}(t)\right)+ \\
& \left.+2 \int_{0}^{t} M^{\prime}\left\|\nabla u_{m}(s)\right\|^{2}\right)\left(\nabla u_{m}(s), \nabla u_{m}^{\prime}(s)\right)^{2} d s+ \\
& +\int_{0}^{t} M\left(\left\|\nabla u_{m}(s)\right\|^{2}\right)\left\|\nabla u_{m}^{\prime}(s)\right\|^{2} d s+c(\epsilon) \int_{0}^{t}\left\|b^{m}\left(u_{m}^{\prime}(s)\right)\right\|_{\Gamma_{1}}^{2} d s+ \\
& +\epsilon \int_{0}^{t}\left\|u_{m}^{\prime \prime}(s)\right\|_{\Gamma_{1}}^{2} d s+c_{12}+\epsilon \int_{0}^{t}\left\|u_{m}^{\prime \prime}(s)\right\|^{2} d s+c(\epsilon) \int_{0}^{t}\|f(s)\|^{2} d s
\end{aligned}
$$

Since $\epsilon$ is arbitrary and $M(s)$ is a $C^{1}$ function and from $(3.13)-(3.16)$, (3.24), we 
conclude that

$$
\int_{0}^{t}\left\|u_{m}^{\prime \prime}(s)\right\|^{2} d s+\left\|\nabla u_{m}^{\prime}(t)\right\|^{2}+\int_{0}^{t}\left\|u_{m}^{\prime \prime}(s)\right\|_{\Gamma_{1}}^{2} d s+\left\|u_{m}^{\prime}(t)\right\|_{p+2, \Gamma_{1}}^{p+2} \leq c_{13}
$$

Jusit mow, from $(3.13)-(3.16)$ and $(3.24)$, taking into consideration that $\left.u\right|_{\Gamma_{0}}=0$, we obtain

$$
\begin{aligned}
& \left(u_{m}\right) \text { is bounded } \operatorname{in} L^{\infty}\left(0, T ; H_{1}(\Omega)\right) \\
& \left(u_{m}^{\prime}\right) \text { is bounded } \operatorname{in} L^{\infty}\left(0, T ; H_{1}(\Omega)\right) \cap L^{\infty}\left(0, T ; L^{p+2}\left(\Gamma_{1}\right)\right) \\
& \left(( u _ { m } ^ { \prime \prime } ) \text { is lbounded } \operatorname { i n } L ^ { 2 } \left(\left(0, T ; L^{2}(\Omega) \cap L^{2}\left(\Gamma_{1}\right)\right)\right.\right. \\
& \left(b^{m}\left(u_{m}^{\prime}\right)\right) \text { is bounded } \operatorname{in} L^{2}\left(0, T ; L^{2}\left(\Gamma_{1}\right)\right)
\end{aligned}
$$

\section{STEP 2: PASSAGE TO THE LIMIT}

Multiplying (3.1) by $\psi \in C^{1}(0, T)$ whith $\psi(T)=0$ and integrating over $(0, T)$, we obtain

$$
\begin{aligned}
& \int_{0}^{t}\left\{\left(u_{m}^{\prime \prime}(t), w_{j}\right)+\left(\nabla u_{m}^{\prime}(t), \nabla w_{j}\right)+M\left(\left\|\nabla u_{m}(t)\right\|^{2}\right)\left(\nabla u_{m}(t), \nabla w_{j}\right)+\right. \\
& \left.+\left(b^{m}\left(u_{m}^{\prime}(t)\right), w_{j}\right)_{\Gamma_{1}}+\left(\left|u_{m}^{\prime}(t)\right|^{p} u_{m}^{\prime}(t)-K^{\prime}\left(u_{m}(t)\right)\left(u_{m}^{\prime}(t)\right)^{2}, w_{j}\right)_{\Gamma_{1}}\right\} \psi(t) d t- \\
& +\int_{0}^{T}\left(K\left(u_{m}(t)\right) u_{m}^{\prime}(t), w_{j}\right)_{\Gamma_{1}} \psi^{\prime}(t) d t+ \\
& +\int_{0}^{T}\left(u_{m}^{3}(t), w_{j}\right) \psi(t) d t=\int_{0}^{T}\left(f(t), w_{j}\right) \psi(t) d t
\end{aligned}
$$

From (3.25), we have subsequence (we denote by the same symbols as original sequence) such that

$$
u_{m} \rightarrow u \text { weakly star in } L^{\infty}\left(0, T ; H_{1}(\Omega)\right)
$$

$$
u_{m}^{\prime} \rightarrow u^{\prime} \text { weakly star in } L^{\infty}\left(0, T ; H_{1}(\Omega)\right) \cap L^{\infty}\left(0, T ; L^{p+2}\left(\Gamma_{1}\right)\right)
$$

$$
u_{m}^{\prime \prime} \rightarrow u^{\prime \prime} \text { weakly in } L^{2}\left(0, T ; L^{2}(\Omega) \cap L^{2}\left(\Gamma_{1}\right)\right)
$$

$$
b^{m}\left(u_{m}^{\prime}\right) \rightarrow \Xi \text { weakly in } L^{2}\left(0, T ; L^{2}\left(\Gamma_{1}\right)\right)
$$


From $(3.27)$ - (3.29), considering that imbedding $H_{1}(\Omega) \rightarrow L^{2}\left(\Gamma_{1}\right)$ is continuous and compact and using Aubing compactness theorem [2], we have

$$
\begin{gathered}
\left|u_{m}^{\prime}\right|^{p} u_{m}^{\prime}, K\left(u_{m}\right) u_{m}^{\prime}, K^{\prime}\left(u_{m}\right)\left(u_{m}^{\prime}\right)^{2} \in L^{q}\left(\Sigma_{1}\right), q=\frac{p+2}{p+1}>1 \\
u_{m} \rightarrow u \text { a.e. on } \Sigma_{1} \text { and } u_{m}^{\prime} \rightarrow u^{\prime} \text { a.e. on } \Sigma_{1}
\end{gathered}
$$

Therefore,

$$
\begin{cases}\left|u_{m}^{\prime}\right|^{p} u_{m}^{\prime} \rightarrow\left|u^{\prime}\right|^{p} u^{\prime}, K\left(u_{m}\right) u_{m}^{\prime} \rightarrow K(u) u^{\prime} & \\ K^{\prime}\left(u_{m}\right)\left(u_{m}^{\prime}\right)^{2} \rightarrow K^{\prime}(u)\left(u^{\prime}\right)^{2} & \text { a.e. on } \Sigma_{1}\end{cases}
$$

\section{STEP 3: $(u, \Xi)$ IS A SOLUTION OF (1.1)}

Letting $m$ tend to infinity in (3.25) and using (3.27) - (3.32) and (3.20)

$$
\begin{aligned}
& \int_{0}^{t}\left\{\left(u_{m}^{\prime \prime}(t), w_{j}\right)+\left(\nabla u^{\prime}(t), \nabla w_{j}\right)+M\left(\|\nabla u(t)\|^{2}\right)\left(\nabla u(t), \nabla w_{j}\right)+\right. \\
& \left.+\left(\Xi(t), w_{j}\right)_{\Gamma_{1}}+\left(\left|u^{\prime}(t)\right|^{p} u^{\prime}(t)-K^{\prime}(u(t))\left(u^{\prime}(t)\right)^{2}, w_{j}\right)_{\Gamma_{1}}\right\} \psi(t) d t- \\
& \quad+\int_{0}^{T}\left(K(u(t)), u^{\prime}(t), w_{j}\right) \psi^{\prime}(t) d t+\int_{0}^{T}\left(u^{3}(t), w_{j}\right) \psi(t) d t=\int_{0}^{T}\left(f(t), w_{j}\right) \psi(t) d t
\end{aligned}
$$

Since $\left\{w_{j}\right\}$ is dense in $H_{1}(\Omega) \cap L^{p+2}(\Gamma)$, we conclude that (2.4) hold.

Only it remains to show (2.5), i.e., $(\Xi(x, t)) \in \varphi\left(u^{\prime}(x, t)\right)$ a.e. $(x, t) \in \Sigma_{1}$. By the Aubin-Lions compactness Lema in [2], we get from (3.28) - (3.29) that

$$
u_{m}^{\prime} \rightarrow u^{\prime} \text { Strongly in } L^{2}\left(0, T ; L^{2}\left(\Gamma_{1}\right)\right)
$$

This implies

$$
u_{m}^{\prime}(x, t) \rightarrow u^{\prime}(x, t) \text { a.e. on } \Sigma_{1}
$$

Thus, for given $\eta>0$, using the theorems of Lusing and Egoroff, we can choose a subset $w \subset \Sigma_{1}$ such that means $(w)<\eta, u^{\prime} \in \Sigma \backslash w$ and $u_{m}^{\prime} \rightarrow u^{\prime}$ uniformly on $\Sigma \backslash w$. Thus, for each $\epsilon>0$, there is a $N>\frac{\epsilon}{2}$ such that

$$
\left|u_{m}^{\prime}(x, t)-u^{\prime}(x, t)\right|<\frac{\epsilon}{2} ; \forall(x, t) \in \Sigma_{1} \backslash w
$$


Then, if $\left|u_{m}^{\prime}(x, t)-s\right|<\frac{1}{m}$, we have $\left|u^{\prime}(x, t)-s\right|<\epsilon$ for all $m>N$ and $(x, t) \in \Sigma_{1} \backslash w$. Therefore,

$$
\underline{b}_{\epsilon}\left(u^{\prime}(x, t)\right) \leq b^{m}\left(u_{m}^{\prime}(x, t)\right) \leq \bar{b}_{\epsilon}\left(u^{\prime}(x, t)\right) ; \forall w>N ;(x, t) \in \Sigma_{1} \backslash w
$$

Sea $\phi \in L^{\infty}\left(\Sigma_{1}\right), \phi \geq 0$, then

$$
\begin{aligned}
\int_{\Sigma_{1} \backslash w} \underline{b}_{\epsilon}\left(u^{\prime}(x, t)\right) \phi(x, t) d \Gamma d t & \leq \int_{\Sigma_{1} \backslash w} b^{m}\left(u_{m}^{\prime}(x, t)\right) \phi(x, t) d \Gamma d t \\
& \leq \int_{\Sigma_{1} \backslash w} \bar{b}_{\epsilon}\left(u^{\prime}(x, t)\right) \phi(x, t) d \Gamma d t
\end{aligned}
$$

Letting $m$ approach $\infty$ in (3.33) and using (3.29), we obtain

$$
\begin{aligned}
\int_{\Sigma_{1} \backslash w} \underline{b}_{\epsilon}\left(u^{\prime}(x, t)\right) \phi(x, t) d \Gamma d t & \leq \int_{\Sigma_{1} \backslash w} \Xi(x, t) \phi(x, t) d \Gamma d t \\
& \leq \int_{\Sigma_{1} \backslash w} \bar{b}_{\epsilon}\left(u^{\prime}(x, t)\right) \phi(x, t) d \Gamma d t
\end{aligned}
$$

Letting $\epsilon \rightarrow 0^{+}$in (3.34), we infer that

$$
\Xi(x, t) \in \varphi\left(u^{\prime}(x, t)\right) \text { a.e. in } \Sigma_{1} \backslash w
$$

and letting $\eta \rightarrow 0^{+}$we get

$$
\Xi(x, t) \in \varphi\left(u^{\prime}(x, t)\right) \text { a.e. in } \Sigma_{1}
$$

This complete the proof.

\section{CONCLUSIONS}

The technique used to find the solution to the generalized system (4.1), it is quite usual, Faedo-Galerkin method and results of Compactness, so unusual is that it applies to problems with terms of Differential Inclusion. Currently, many researchers are under doomed to study differential equations with Inclusion. What would also be interesting to see is the study of asymptotic behaviour. This paper will serve as guidance for the study of equations, which may be affected with terms of Differential Inclusion on the boundary. 


\section{REFERENCES}

[1] Jong Yeoul Park and Sun Hye Park - Solutions for a Hyperbolic System with boundary differential inclusion and nonlinear second-order boundary damping. Electronic Journal of Differential Equations Vol 2003 (2003) No 80, pp. 1-7.

[2] J.L. Lions - Quelques méthodes de résolution de problémes aux limites non linéares. Dunod Gauthiers Villars, Paris, 1969.

[3] M. Miettinem - A parabolic hemivariational inequality, Nonlinear Anal. 26(1996), pp. 725-734.

[4] M. Miettinem and P.D. Panagiotopoulos. - On parabolic hemivariational inequalities and applications, Nonlinear Anal. 35(1999), pp. 885-915.

[5] J. Rauch - Discontinuos semilinear differential equations and multiple value maps, Proc. Amer. Math. Soc. 64 (1977), pp. 277-282.

[6] G.G. Doronin, N.A. Lar kin and A.J. Souza - A hyperbolic problem with nonlinear second order boundary damping, Electronic J. Diff. Eqs. 1998 (1998) No 28, pp. $1-10$. 